\title{
Electrolyte Level Changes in Acute Myocardial Infarction Patients as Compared to Healthy Individuals in Khan Younis Governorate, Gaza Strip
}

\author{
Lamia Fasail Abu Marzoq*, Wafaa Hamad Jaber, Dina K. Halaid Azzam \\ Medical Sciences Department, University College of Science and Technology, Gaza Strip, Palestine \\ Email address: \\ 1.marzoq@gmail.com (L. F. A. Marzoq)
}

\section{To cite this article:}

Lamia Fasail Abu Marzoq, Wafaa Hamad Jaber, Dina K. Halaid Azzam. Electrolyte Level Changes in Acute Myocardial Infarction Patients as Compared to Healthy Individuals in Khan Younis Governorate, Gaza Strip. Advances in Biochemistry. Vol. 4, No. 2, 2016 pp. 9-15. doi: $10.11648 /$ j.ab.20160402.11

Received: March 8, 2016; Accepted: March 16, 2016; Published: April 5, 2016

\begin{abstract}
Acute myocardial infarction (AMI) is one of the leading causes of morbidity and mortality across the world. Serum electrolytes changes in AMI have not been studied in Khan Younis Governorate-Palestine. Hence, this study was undertaken to investigate any changes in the serum electrolytes with special attention to serum sodium, potassium, calcium, magnesium and chloride in cases of AMI. Aim: To determine the pattern of changes of serum electrolytes AMI patients and then compare with non AMI (i.e. healthy persons). Materials and Method: The study consisted of a hundred people divided equally into study group and control group. Samples were taken from the Central Heart Unit in Nasser Hospital - Khan Younis. The study group comprised cases of confirmed diagnosis of recent onset of AMI. Clinical data was collected using interviewing questionnaires. The first paragraph of the questionnaire was for personal data which included age, sex and education level. The blood samples of both groups were automatically analyzed for serum electrolytes $\left(\mathrm{Na}^{+}, \mathrm{K}^{+}, \mathrm{Cl}^{-}, \mathrm{Ca}^{+}\right.$and $\mathrm{Mg}^{+}$) where the latter was analyzed manually. The cases were taken from AMI patients within 48 hours of admission. Results: The mean of age of controls was $47.18 \pm 64$ while that of cases was $56.34 \pm 12.84$. Significant low level of calcium was found in AMI patients while the level observed for magnesium was high. The levels of potassium and magnesium in AMI patients with hypertension was observed to be low with p-value $<0.05$. Moreover, potassium levels were also low in AMI patients with diabetes with $\mathrm{p}$-value $<0.05$. The changesin electrolytes werenot statistically significant in association with smoking. Conclusion: The decrease in sodium level was observed only in patients with AMI compared with healthy persons.
\end{abstract}

Keywords: Acute Myocardial Infarction, Serum Chloride, Serum Potassium, Serum Sodium, Serum Magnesium and Serum Calcium

\section{Introduction}

Acute Myocardial Infarction (AMI) is a dreadful complication of cardiovascular disease causing increasing mortality worldwide. The prevalence of Myocardial Infarction (MI) is more in the middle income world (WHO, 2004) and it is well known that males are more commonly affected than females. It is a disease commonly known as a heart attack. This disease occurs when blood flow stops to part of the heart causing damage to the heart muscle thus increasing myocardial metabolic demand, decreasing delivery of oxygen and nutrients to the myocardium via the coronary circulation. The most common symptom is chest pain or discomfort which may travel into the shoulder, arm, back, neck or jaw. Often, it is in the center or left side of the chest and lasts for more than a few minutes. MI is one of the most common disease diagnosed in hospitalized patients in industrialized countries. It is a growing cause of death worldwide. Sudden cardiac death occurs worldwide at a rate of 3 million per year (Jeldsen K. K., 2010).

Electrolyte is the medical term for salt that is found in the blood and other body fluids. The electrolyte levels, being modifiable, play an important role in altering the prognosis of MI patients. These electrolyte level changes have been reported to be used to monitor AMI. There are several common electrolytes found in the body, each serving a 
specific and important role, but most are in some part responsible for maintaining the balance of fluids between the intracellular (inside the cell) and extracellular (outside the cell) environments. This balance is critically important for issues like hydration, nerve impulses, muscle function and $\mathrm{pH}$ level. The major electrolytes in the body are Potassium, Sodium, Magnesium, Calcium and Chloride. Serum Sodium, Potassium and Chloride are considered to be the major determinants of electrophysiological properties of myocardial membrane.

Potassium $(\mathrm{K})$ is a main component of cellular fluid. This positive electrolyte helps to regulate neuromuscular function and osmotic pressure, Approximately $98 \%$ of this electrolyte is intracellular. Its main regulation is by the renal excretion and shift between the intracellular and extracellular compartments. Potassium is one of the electrolytes that play an important role in cardiac disease specially AMI.
Hypokalemia defined as serum Potassium levels <3.5 $\mathrm{mmol} / \mathrm{l}$ is portrayed as a determinant of excessive morbidity in such patients, particularly malignant ventricular arrhythmia. Several studies have shown association between hypokalemia, even mild hypokalemia with increased occurrence of cardiac arrhythmias in AMI patients (Nordrehaug J. E., 1985).Hypokalemia in patients with AMI is thought to predict increased in-hospital morbidity, particularly arrhythmias and mortality (Xianghua F. et al., 2000).

Sodium $(\mathrm{Na})$ is the most abundant extracellular cation, a positively charged electrolyte that helps to balance fluid levels in the body and facilitates neuromuscular functioning. Serum Sodium imbalance has been also recorded in early phase of AMI in some studies (Goldberg A.et al., 2004). Hyponatremia defined as serum Sodium concentration $<135$ $\mathrm{mmol} / \mathrm{l}$ is relatively common among patients hospitalized with AMI (Tada Y. et al., 2011).

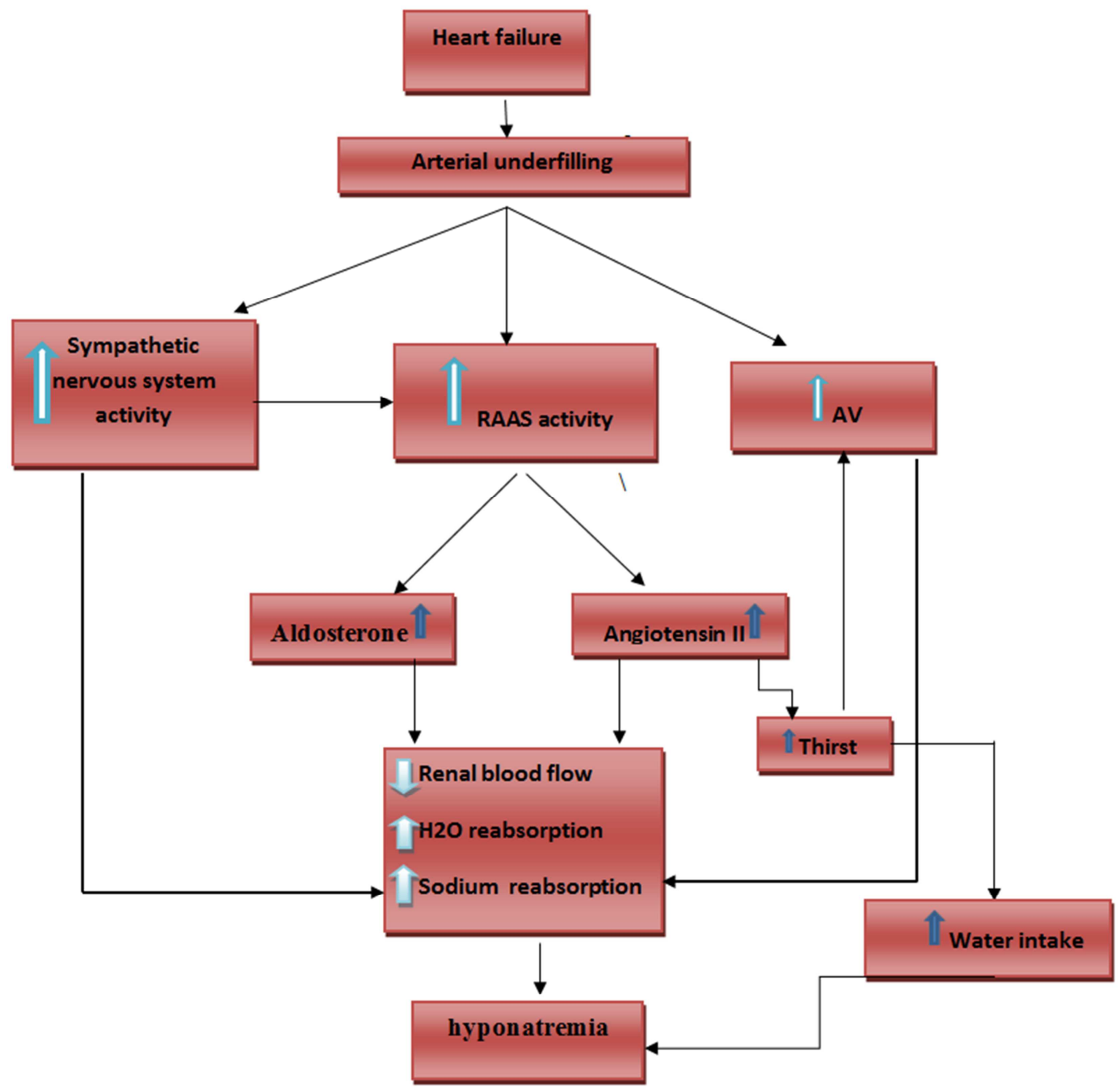

Figure 1. Mechanism of hyponatremia in patient with heart failure (RAAS: Renin-angiotensin-aldosterone system; AVP: Argenine-vasopressin).

Magnesium $(\mathrm{Mg})$ is the second most abundant intracellular cation and it is vital for more than 300 enzymatic reactions which are involved in various metabolic processes in the body, but still, it is often a parameter which is overlooked by the clinicians (Shaikh S. and Karira K. A., 2011). Magnesium is a cardio protective element because of its $\beta$-adrenoreceptor blocking action, antiplatelet action and inhibitory effect on the cardiac conducting system (Myoishi M. and Kitakaze M., 2005). The ion-stabilizing effect of Magnesium helps in maintaining stable intra and extracellular concentrations of 
other electrolytes. Studies have documented significant alterations in $\left(\mathrm{Mg}^{2+}\right)$ and other electrolytes in patients with AMI (Maciejewski P. et al., 2003).

One of the earliest studies that showed a beneficial effect of Magnesium in the pre-thrombolytic era was conducted by
Rasmussen at a hospital in Copenhagen that was served by a highly efficient ambulance service such that patients with AMI were admitted in $<1$ hour from the onset of chest discomfort (Rasmussen H., 1995).

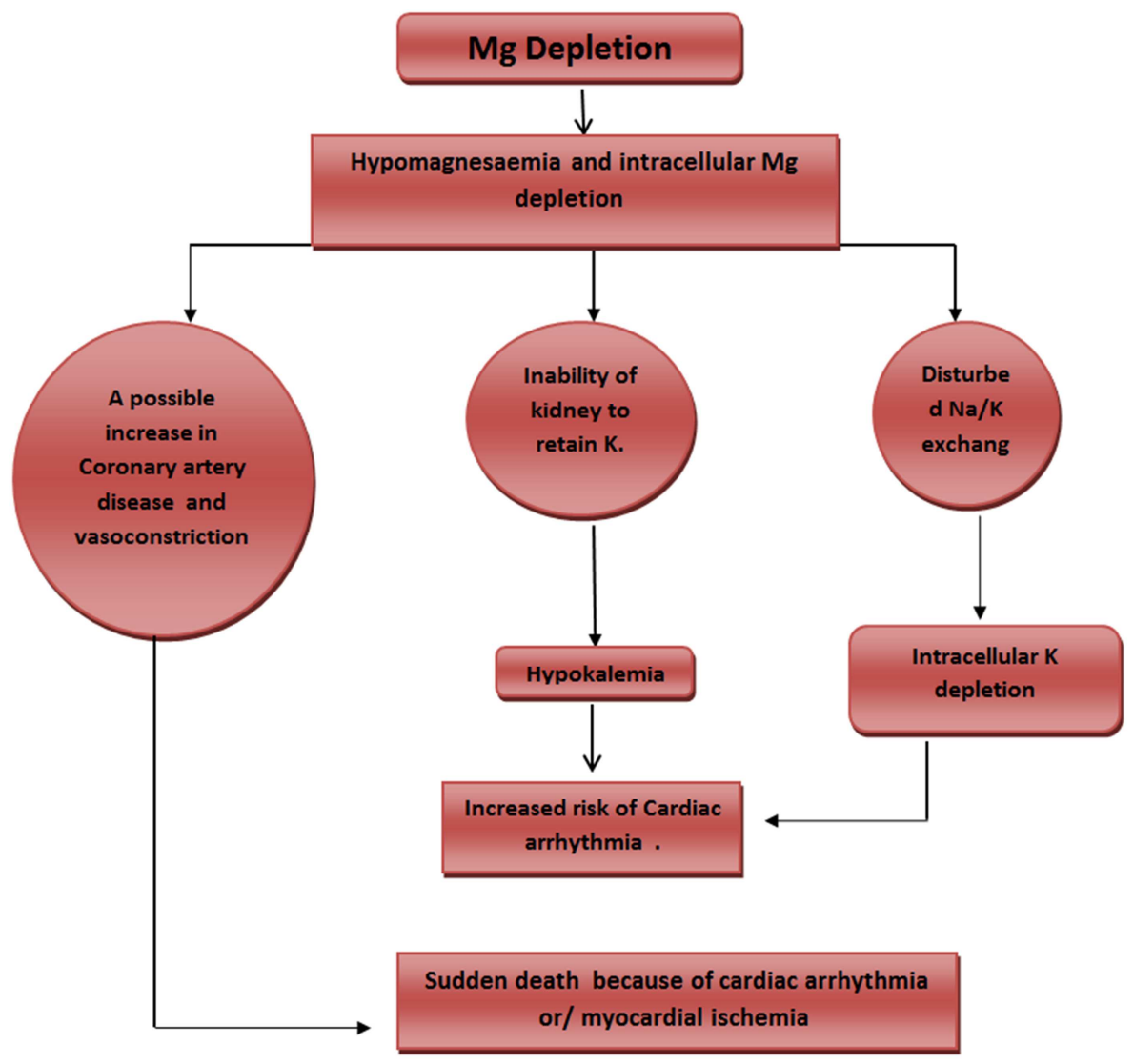

Figure 2. Mechanism of hypomagnesemia in patient with heart failure.

Calcium (Ca) ions play a vital role in the sequence of excitation-contraction of the cardiac muscle fibers and they are essential in both the cardiac and systemic vasculature (Lehmann G. et al., 2000). Furthermore, hypocalcemia impairs myocardial contractility and there are several reports of congestive heart failure caused by severe hypocalcemia, while long-standing hypocalcemia has been implicated in the pathogenesis of cardiomyopathy. Moreover, coronary spasm due to hypocalcemia has been reported as the most likely mechanism of chest pain in young patients mimicking AMI.

Chloride $(\mathrm{Cl})$ is the major negatively-charged ion (anion). Chloride is primarily found in extracellular fluid and works closely with Sodium to maintain proper balance and pressure of the various fluid compartments of the body (blood, inside cells and the fluid between cells). It is also vitally important for maintaining proper acidity in the body, passively balancing out the positive ions of blood, tissue and organs.

Several mechanisms interact to produce these electrolyte disturbances. The decrease in cardiac output leads directly to a reduction in renal blood flow, with impairment of renal excretion of water and electrolytes. It also causes the activation of several neurohormonal responses which affect both cardiovascular homeostasis and electrolyte balance.

\section{Objectives}

\subsection{General Objective}

To determine the pattern of changes of serum electrolytes among post AMI patients as well as to compare the electrolytes levels among both MI and non-MI patients.

\subsection{Specific Objective}

1. To find out the pattern changes of serum electrolytes in case group as compared to control group.

2. To detect the presence of electrolyte changes in cases with AMI and in controls without MI.

3. To compare the electrolyte levels among cases and controls.

\subsection{Problem Statement}

The incidence of AMI is high and it is the leading cause of death in the elderly also. According to WHO 14 million people die annually in the world (WHO, 2011).

Magnesium is very useful for the treatment of AMI. 
Magnesium promotes the vasodilation and prevents from vasospasm. It has antiarrhythmic activity and also plays a great role in producing myocardial energy. It was reported that intravenous administration of the right amount of Magnesium decreases mortality and also improves the clinical outcomes in patients with AMI. Magnesium has seen beneficial when it was used as a primary therapy in AMI treatment. Magnesium can be used as first line treatment of AMI patients who are not candidates of fibrinolysis therapy like people with uncontrolled hypertension, recent stroke and current bleeding

Hypomagnesaemia is a common clinical finding in hospitalized patients and can cause hypocalcaemia, cardiac arrhythmias, muscular weakness, and hypokalemia

\subsection{Significance}

Myocardial infarction is a major cause of death and disability and in Palestine as in the rest of the world. AMI is considered the main cause of death among males $15-59$ years old. Mortality rate for MI is $18.7 \%$ in males and $7.7 \%$ in females worldwide.

AMI is being a health problem of paramount importance in terms of frequency, seriousness, social and economic costs, amenability to medical intervention and priority-ranking by policy makers and the community.

AMI mortality in non-hospitalized cases is very high in the first two hours, implying the need to combine causes of death statistics and hospitalization data to obtain incidence figures.

\subsection{Hypotheses}

- Hypomagnesaemia in AMI increases the risk of tachycardia.

- Patients with AMI have low level of Sodium and Potassium.

\section{Materials and Methods}

\subsection{Study Population}

The study was case control study and started in July 2015 and was completed in October 2015. The population of the study comprised of 50 AMI case group with age and sex matched with healthy individual as control group.

\subsection{Ethical Consideration}

1. A formal letter of approval to start the study was obtained from the graduate committee of the Medical Sciences Department of the University College of Sciences and Technology, Khan Younis.

2. Anofficial approval letter was obtained from the human resources development of the Palestinian Ministry of Health (MoH).

3. An official approval letter was be obtained from the Central Heart Unit at Nasser Hospital in Khan Younis to conduct the study and to perform the required biochemical analysis in their laboratory.

\subsection{Sampling Sites and Sample Size}

Fifty sample were taken from the Central Heart Unit at Nasser Hospital. Sampling technique was used to select the study AMI patients who were attending the clinic. Another fifty samples were taken from healthy persons and used as control.

\subsection{Inclusion and Exclusion Criteria}

\subsubsection{Inclusion}

Selection of patients with AMI was according to doctor's diagnosis through signs and symptoms in a given period (6 hours). In the control cases, these samples were not suffering from hypertension, renal failure, diarrhea, vomiting, AMI and were not smokers.

\subsubsection{Exclusion}

Patients with renal insufficiency were excluded based on serum creatinine levels as well as hyperglycemic MI patients. Known cases of liver failure, chronic vomiting, diarrhea patient, malignancy, adrenal insufficiency and hypertensive patients were also excluded based on their history.

\subsection{Data Analysis}

The data was analyzed by using the Statistical Package of Social Sciences (SPSS) software, version 18. The values were expressed as mean and standard deviation. The independent $\mathrm{T}$-test was performed to compare the mean values between the cases and the controls.

A significant result means that the $\mathrm{p}$-value tests is $<0.05$. The confidence intervals (CI) was reported as $95 \%$.

\subsection{Materials and Tools}

Table 1. Materials and Tools.

\begin{tabular}{|c|c|c|}
\hline Disposables & Instruments & Reagents \\
\hline $\begin{array}{l}\text { Syringes } 5 \mathrm{ml} \text {, } \\
\text { Needles }\end{array}$ & Centrifuge & Electrolyte's Reagent \\
\hline $\begin{array}{l}\text { Cotton, Alcohol, } \\
\text { Gloves and Tubes }\end{array}$ & Spectrophotometer & Magnesium Kit \\
\hline - & Electrolyte analysis instrument & - \\
\hline
\end{tabular}

\subsection{Questionnaire Interview}

A meeting interview was used for filling in questionnaire. The questionnaire consisted of two parts: socio-demographic data (name, age, sex, education status, etc.) and clinical data (family history of AMI, AMI duration, type of hyperelectremia drugs, etc.).

\section{Results}

\subsection{Study Population}

The present work included 100 subjects, classified into two groups: 50 case patients with AMI from the Central Heart Unit at Nasser Hospital and 50 control of healthy persons. The mean age of the controls was $47.18 \pm 4.64$ years while the mean of age of the cases $56.34 \pm 12.84$ years. The 
distribution of the subjects according to gender was 68 male and 32 female. Out of the 50 cases, 28 cases were hypertensive and 16 cases were diabetic.

\subsection{Sodium and Potassium Levels in the Case and Control Groups}

The results of the study showed that there was a statistically significant difference between the case and control group in serum Sodium ( $p$-value $=0.00$ ). However, there was no significant difference between control and case group in serum Potassium level ( $\mathrm{p}$-value $=0.626$ ), as shown in Table 2 below.

Table 2. Comparison of Sodium and Potassium serum between case and control.

\begin{tabular}{llll}
\hline Serum $(\mathbf{m m o l} / \mathbf{L})$ & Case & Control & p-value \\
\hline Sodium $\mathrm{Na}^{+}$ & $123.28 \pm 16.6$ & $134.34 \pm 11.02$ & 0.00 \\
Potassium K & $4.324 \pm 1.15$ & $4.467 \pm 1.72$ & 0.626 \\
\hline
\end{tabular}

\subsection{Other Electrolytes Levels in the Study Groups of Both Case and Control}

The results of the study showed that there was a statistically significant difference between the case and control in levels of serum $\mathrm{Mg}, \mathrm{Ca}+2$ and $\mathrm{Ca}+2$ total, with $\mathrm{p}$ values $<0.001$. There was no statistically significant difference in serum Chloride, as shown in Table 3 below.

Table 3. Comparison of other electrolytes between case and control.

\begin{tabular}{llll}
\hline Serum $(\mathbf{m m o l} / \mathbf{L})$ & Case & Control & p-value \\
\hline Chloride $\mathrm{Cl}^{-}$ & $103.278 \pm 9.22$ & $105.188 \pm 8.06$ & 0.273 \\
Calcium $\mathrm{Ion} \mathrm{Ca}^{2+}$ Ion & $3.753 \pm 0.710$ & $4.243 \pm 0.572$ & 0.00 \\
Magnesium $\mathrm{Mg}^{2+}$ & $4.729 \pm 4.043$ & $2.537 \pm 1.290$ & 0.001 \\
Calcium Total Ca Total & $7.508 \pm 1.411$ & $8.502 \pm 1.125$ & 0.00 \\
\hline
\end{tabular}

\subsection{Relationship Between AMI and Demographical Variables}

\subsubsection{Mean and SD of Electrolyte Levels in Cases with and Without Hypertension}

The results of the study showed that there is a statistically significant difference between the hypertensive cases and non-hypertensive in level of serum Potassium and Magnesium where is $p$-value $<0.05$. There was no statistically significant difference in other electrolytes, as shown in Table 4 below.

Table 4. Mean and SD of electrolyte levels in cases with\& without hypertension.

\begin{tabular}{llll}
\hline Serum $(\mathbf{m m o l} / \mathbf{L})$ & Hypertensive & Non-hypertensive & p-value \\
\hline Sodium $\mathrm{Na}^{+}$ & $121.9 \pm 19.4$ & $124.9 \pm 12.4$ & 0.59 \\
Potassium $\mathrm{K}^{+}$ & $4.4 \pm 1.4$ & $4.1 \pm 0.49$ & 0.05 \\
Chloride $\mathrm{Cl}^{-}$ & $101.63 \pm 10.96$ & $105.36 \pm 5.98$ & 0.212 \\
Calcium Total Ca Total & $7.74 \pm 1.5$ & $7.205 \pm 1.14$ & 0.10 \\
Calcium Ion $\mathrm{Ca}^{2+}$ Ion & $3.87 \pm 0.788$ & $3.601 \pm 0.580$ & 0.1 \\
Magnesium $\mathrm{Mg}^{2+}$ & $4.673 \pm 3.38$ & $4.80 \pm 4.879$ & 0.05 \\
\hline
\end{tabular}

\subsubsection{Mean and SD of Electrolyte Levels in Cases with and Without Diabetic}

The results of the study revealed that there was statistically significant difference between the diabetic and non-diabetic cases in the level of potassium with $p$-value $<0.005$. On the other hand, for other electrolytes there, was no statistically significant as shown in Table 5.

Table 5. Mean and SD of electrolyte levels in cases with and without diabetic.

\begin{tabular}{llll}
\hline Serum $(\mathbf{m m o l} / \mathbf{L})$ & Diabetic & Non-diabetic & p-value \\
\hline Sodium $\mathrm{Na}^{+}$ & $126.6 \pm 15.2$ & $121.7 \pm 17.2$ & 0.69 \\
Potassium $\mathrm{K}^{+}$ & $4.652 \pm 1.84$ & $4.169 \pm 0.593$ & 0.005 \\
Chloride $\mathrm{Cl}^{-}$ & $104.14 \pm 7.84$ & $102.87 \pm 9.89$ & 0.502 \\
Calcium Total Ca Total & $7.871 \pm 1.32$ & $7.33 \pm 1.4$ & 0.62 \\
Calcium Ion $\mathrm{Ca}^{2+}$ Ion & $3.93 \pm 0.658$ & $3.66 \pm 0.727$ & 0.58 \\
Magnesium $\mathrm{Mg}^{2+}$ & $4.575 \pm 3.94$ & $4.801 \pm 4.14$ & 0.67 \\
\hline
\end{tabular}

\subsection{Mean and SD of Electrolyte Levels in Cases with and Without Smoking}

The results of the study showed that there was not a statistically significant difference between smokers and nonsmokers in the levels of all electrolytes p-value $>0.05$ as shown in Table 6.

Table 6. Mean and SD of electrolyte levels in cases with and without smoking.

\begin{tabular}{llll}
\hline Serum $(\mathbf{m m o l} / \mathbf{L})$ & Smoker & Non-Smoker & p-value \\
\hline Sodium $\mathrm{Na}^{+}$ & $128.9 \pm 8.99$ & $121.06 \pm 18.4$ & 0.15 \\
Potassium $\mathrm{K}^{+}$ & $4.07 \pm 0.35$ & $4.42 \pm 1.33$ & 0.08 \\
Chloride $\mathrm{Cl}^{-}$ & $107.1 \pm 4.5$ & $101.7 \pm 10.1$ & 0.08 \\
Calcium Total Ca Total & $7.44 \pm 1.4$ & $7.5 \pm 1.4$ & 0.77 \\
Calcium Ion $\mathrm{Ca}^{2+}$ Ion & $3.72 \pm 0.74$ & $3.76 \pm 0.7$ & 0.73 \\
Magnesium $\mathrm{Mg}^{2+}$ & $4.09 \pm 3.05$ & $4.97 \pm 4.38$ & 0.15 \\
\hline
\end{tabular}

\section{Discussion}

Electrolyte imbalances are fairly common in acute phase of MI patients when we studied changes of levels of electrolytes in AMI, it was included 100 subjects, was classified into two groups 50 case patients with AMI and 50 Control healthy persons. The study was studied group of electrolyte as Sodium, Potassium, Chloride, Calcium total, Calcium ion and Magnesium.

\subsection{Relationship Between Electrolytes and Case}

From this study, we can conclude that there was statistically significant decreased in serum Sodium in study compared to normal healthy control group $(\mathrm{p}$-value $=0.00)$ in Table 2, which was similar to finding was observed by Amrita Vamne et al. (2015) who reported hyponatremia in cases of AMI patient when compared with control, decrease in sodium level was due to hypoxia and ischaemia, which increase the permeability of sarcolemma to sodium ,but there was no significant difference between control and case in serum potassium level $(\mathrm{p}$-value $=0.626)$ in Table 2 . Our study also supported the hypothesis that the alterations in the 
electrolyte levels could prompt the pathological events in coronary heart diseases. On the other hand, the opposite finding was observed by Nordrehaug J. E. (1985) who showed significant lower of serum Potassium among AMI cases.

The decrease of Magnesium level is common in AMI patient. Our study, however, showed that there was increase in Magnesium level in AMI patients as compared to the control with $\mathrm{p}$-value $=0.001$ in Table 3 . The decrease of Magnesium level we suggested due to admission of Magnesium as the first step of treatment.

Our study also showed that there was decrease in total serum Calcium and serum ionized Calcium with $\mathrm{p}$-value $=$ 0.00 it is similar to the study of Ramasamy R. et al.(2013) who reported significantly lowering in AMI with p-value < 0.001.Chloride Ion had no significant value in this study as similar as to Mati E. et al. (2012) study.

\subsection{Mean and SD of Electrolyte Levels in Cases with and Without Hypertension}

The decrease in Potassium level is common in hypertensive patients of AMI. Our study showed that there increase of potassium level in hypertensive patients when compared with non-hypertensive $p$-value $=0.05$ it is come as opposite of Vamne A. (1985) study who reported that there was no effect value for potassium on hypertension, decrease of potassium level was influenced by lowering in Magnesium level.

The decrease in magnesium level is common in hypertensive case than non-hypertensive case with $\mathrm{p}$-value $=$ 0.055 which is similar to Kiranmai et al. (2014) study. This study showed decrease in $\mathrm{Mg}^{2+}$ in hypertensive cases, low level of magnesium predisposes to increase in arterial pressure as $\mathrm{Mg}^{+2}$ acts peripherally to produce peripheral vasodilation and hence fall in blood pressure.

\subsection{Mean and SD of Electrolyte Levels in Cases with and Without Diabetic}

The present study shown that serum potassium is the only electrolyte that give lowering in diabetic patients when compared with non-diabetic patients $(p$-value $=0.005)$. This is opposite to the findings of Vamne A. (2015) study. The increase of Potassium level in AMI patient is due to diabetes mellitus disease due to disorder of insulin level.

Our study also showed that hypertension and diabetes mellitus is common in case with AMI with p-value $=0.00$ as compared with controls. Hypertension regard as risk factor for AMI disease.

The present study showed that there was no statistically significant difference for smoking when compared to control.

\section{Conclusions}

From this study we concluded that hyponatremia was observed in patients with AMI with statistical significance. There is statistically significant increase in Magnesium and that is due to admission of Magnesium as the first step of treatment, before taking sample.

There is statistically significant decrease in serum Magnesium levels in AMI with hypertensive when compared to cases without hypertension and lowering of serum Potassium level in hypertensive cases with AMI, with no significant change in $\mathrm{S} . \mathrm{Ca}^{+2}, \mathrm{~S} . \mathrm{Na}^{+}$and $\mathrm{S} . \mathrm{Cl}^{+}$levels. Low Magnesium levels may be a cause or effect of hypertension. Maintaining the normal serum levels of Magnesium can prevent the development of hypertension.

Hyponatremia is indicator of AMI. Serum Sodium level is prognostic indicators, i.e, rise in Sodium levels after initial fall was indicative of clinical improvement. Therefore, estimation of Sodium and Potassium levels in AMI patients can help assess their prognosis.

\section{Recommendations}

1. According to the results of this and previous studies, we recommend routine serum Magnesium determination and more attention to hypomagnesaemia patients to prevent future complication.

2. Estimation of serum electrolyte is good for diagnosis and prognosis of AMI.

3. Maintaining the normal serum levels of Magnesium can prevent the development of hypertension.

4. We should taking the sample as soon as the patient enters the hospital before any admission.

5. The estimation of serum Sodium level in patients with AMI should be done as early as possible on arrival of the patients in emergency department.

\section{References}

[1] Alexander Goldberg, Haim Hammerman, Sirouch Petcherski, Alexander Zdorovyak, Sergey Yalonetsky, Michael Kapeliovich, Walter Markiewicz, Doron Aronson (2004).

[2] Amrita Vamne, Swati Pathak, Ramesh Chandra Thanna, Rekha Choudhary, (2015), Index Medical College Hospital \& Research Centre, Indore, M. P., India.

[3] Aziz F, Doddi S, Penupolu S, Del Castillo D, Raza W and Kallu S (2011).

[4] P. Kiranmai, N. V. Lakshmi (2014) Comparative Study of Serum Magnesium, Calcium, Potassium and Sodium Levels in Diabetics and Hypertensives with Acute Myocardial Infarction.

[5] Esha Mati, Krishnamurthy N, Ashakiran S, Sumathi M E, Raghavendra Prasad (2012) Departments of Biochemistry, Medicine, Sri Devaraj Urs Medical College, Kolar.

[6] Goldberg A, Hammerman H, Petcherski S, Zdorovyak A Yalonetsky S and Kapeliovich M (2004). The American Journal of Medicine 2004; 117(4): 242-248.

[7] Hadeel Rashid Faraj (2015) Clinical study of some electrolytes (sodium, chloride and potassium) with patients in acute coronary syndrome (ACS) ISSN: 2319-7706. 
[8] Jan Erik Nordrehaug (1985) Malignant arrhythmia in relation to serum potassium in acute myocardial infarction.

[9] Jeldsen K K. (2010) Hypokalemia and sudden cardiac death, Exp Clin Cardiol.

[10] Krishnamurthy N, Ashakiran S, Sumathi M E, Raghavendra (2012). Prasad 1Departments of Biochemistry, Medicine, Sri Devaraj Urs Medical College, Kolar.

[11] Lehmann G, Deisenhofer I, Ndrepepa G, Schmitt C. (2000). ECG changes in a 25- year-old woman with hypocalcemia due to hypoparathyroidism. Hypocalcemi mimicking acute myocardial infarction. Chest 2000; 118 (1).

[12] Mac Donald J E, Struthers A D. (2014) Cardio 2004; 43(2):155-161.

[13] Maciejewski P, Bednarz B, Chamiec T, Górecki A, Łukaszewicz R, Ceremuzynski L. (2003 Nov). Acute coronary syndrome: potassium, magnesium and cardiac .arrhythmia. Kardiol Pol; 59(11).

[14] Munesh Tomar, Sitaraman Radhakrishnan And Savitri Shrivastava (2008).

[15] Myoishi M, Kitakaze M. (2005 Feb) A role of magnesium: magnesium in the therapy for cardiovascular diseases. Clin Calcium.; 15(2): 265-270.

[16] Opie LH. Mechanisms of cardiac contraction and relaxation. In: Braunwald E (ed). Heart disease: a textbook of cardiovascular medicine. WB Saunders, Philadelphia, 1996.
[17] Prognostic Implication of Hyponatremia in Setting of Myocardial Infarction. Chest 1; 140(4 Meeting Abstracts): 986A-986A.

[18] Rasmussen HS. (1988) Justification for intravenous magnesium therapy in acute myocardial infarction. Magnes Res; 1(1-2): 59-73.

[19] Ramesh Ramasamy, Sathish Babumurugaiyan, Niranjan Gopal, Rachel Shalini (2013) Institute Pillayarkuppam Puducherry - 605 402, India

[20] Shaikh S, Karira KA (2011), Magnesium deficiency in heart failure patients withdiabetes mellitus. J Pak Med Assoc; 61(9)

[21] Tada Y, Nakamura T, Funayama H, Sugawara Y, Ako J, Ishikawa S, Momomura S.(2011), Early development of hyponatremia implicates short and long term outcomes in ST elevation acute myocardial infarction. Circ J 2011; 75: 19271933.

[22] Taysir S Garadah, Salah Kassab, Jamal Golbahar, Bahrain Med Bull 2011; 33(1).

[23] Vinod Wali and Singi Yatiraj. (2014) Study of Serum Sodium and Potassium in Acute Myocardial Infarction. DOI: 10.7860/JCDR.

[24] Xianghua F, Peng Q, Yanbo W, Shigiang L, Weize F, Yunfa J.(2010)The relationship between hypokalemia at the early stage of acute myocardial infarction and malignant ventricular arrhythmia; Heart 96: 196. 